\title{
Relevance of human fat distribution on lipid and lipoprotein metabolism and cardiovascular disease risk
}

Marie-Eve Piché1,2, Senthil K Vasan², Leanne Hodson², Fredrik Karpe ${ }^{2,3}$

${ }^{1}$ Quebec Heart and Lung Institute, Laval University, Canada

20xford Centre for Diabetes, Endocrinology and Metabolism, University of Oxford, Churchill Hospital, UK

${ }^{3}$ NIHR Oxford Biomedical Centre, Oxford University Hospital Trust and University of Oxford, Oxford, UK

Abstract: 201

Manuscript: 2534

References: 81

Figures and tables: 2

\section{Address for correspondence}

Prof Fredrik Karpe

Oxford Centre for Diabetes, Endocrinology and Metabolism,

University of Oxford, Churchill Hospital

Oxford OX3 7LE, UK

Email: Fredrik.Karpe@ocdem.ox.ac.uk 


\section{Abstract}

\section{Purpose of review}

Upper body abdominal and lower body gluteofemoral fat depot masses display opposing associations with plasma lipid and lipoprotein and cardiovascular disease (CVD) risk profiles. We review developments on adipose tissue (AT) fatty acid (FA) metabolism in the context of body fat distribution and how that might be related to adverse lipid and lipoprotein profiles and CVD risk.

\section{$\underline{\text { Recent findings }}$}

Recent data have confirmed the paradoxical relationship of upper abdominal and lower body gluteofemoral adiposity and CVD risk. Mechanistically this is likely to reflect the different ways fat depots handle lipid storage and release, which impacts directly and indirectly on lipid and lipoprotein metabolism. The upper body enhances immediate fat storage pathway with rapid uptake of dietary-derived FAs, whereas the lower body fat depot has a reduced lipid turnover accommodating a slower fat redistribution. Body fat distribution and the fat depots' ability to undergo appropriate expansion when fat storage is required, rather than overall body fatness, appear as the important determinant of metabolic health.

\section{Summary}

A focus on fat distribution in overweight people, preferably using precise imaging methods, rather than quantifying total body fatness, is likely to provide the medical community with better tools to stratify and treat patients with obesity-related complications.

Key words: Body fat distribution, adipose tissue expandability, adipose tissue metabolism, lipidlipoprotein profile, cardiovascular risk 


\section{Introduction}

Although obesity per se is positively associated with cardiovascular disease (CVD), this relationship is complex due to several independent associations with CVD risk markers such as hypertension, hyperlipidaemia, insulin resistance or even type 2 diabetes. It is also recognised that total body fatness can have divergent associations with CVD dependent of fat distribution. The recognition of the fact that different fat depots handle lipid metabolism in very different ways [1] and its consequence on whole body lipid and lipoprotein homeostasis will be reviewed in this article. We will pursue the argument that obesity per se is a less important determinant of metabolic dysfunction, instead it is likely to be body fat distribution and the regional fat depots capability to accommodate adequate fat storage that determines metabolic health [2]. 


\section{Fat depots: an overview}

The major compartments for dedicated fat storage in the human body includes the subcutaneous adipose tissue (SAT) depot, defined as upper body abdominal SAT (aSAT) and the lower body gluteofemoral fat depots, which together account for $>80 \%$ of overall body fat mass [3]. Additionally, the intra-abdominal depots which includes visceral adipose tissue (VAT), composed of two major compartments (omental and mesenteric) represents approximately $10 \%$ of overall body fatness in women whereas this proportion may reach up to $25 \%$ in men [4]. Closer examination of the aSAT depot has led to the identification of two anatomically and biologically distinct compartments: a superficial layer of SAT (sSAT) and a deeper SAT (dSAT) compartment, separated anatomically by Scarpa's fascia [5-7]. The adverse effects of excessive 'upper body adiposity' has often been linked to VAT, and the role of aSAT in the regulation of metabolic health has been less recognized until recently. The conventional anthropometric measurement of abdominal obesity is waist circumference (WC) but this surrogate marker does not distinguish between aSAT and VAT. Precise imaging techniques such as computed tomography (CT) scan, magnetic resonance imaging (MRI) and dual energy X-ray absorptiometry (DEXA) provide better estimates of fat depot masses, particularly in the abdominal region.

\section{Upper body abdominal vs lower body gluteofemoral fat depots}

Epidemiological studies have shown that upper and lower body adiposity measured using WC and hip circumference (HC) have paradoxical effects on metabolic health and CVD events and these effects are independent of total body fatness measured with body mass index (BMI) [8-10]. Using DEXA body composition measurements of 4,950 participants in the Oxford Biobank, we have recently shown that upper body fat depots (android and visceral fat) are associated with increased risk of hypertriglyceridaemia, impaired fasting glucose, hypertension and insulin resistance, while lower body fat depots (gynoid and leg fat) have opposite associations when adjusted for total body fat mass [11]. Compared with conventional anthropometry it was very 
clear that precise instruments of quantifying fat depot masses enhanced the ability to observe the paradoxical associations between regional fat masses.

\section{Upper body abdominal fat depots}

Large cohort studies such as the Framingham Heart Study and the Jackson Heart Study using CTimaging, have shown that excess VAT is a predictor of CVD, independently of total body fat mass or SAT [12-15]. In a 10-year longitudinal study of Japanese men, cases developing coronary heart disease (CHD) were approximately 22\% more VAT than controls [16]. Adverse effects like insulin resistance or dyslipidaemia dependent on abdominal adiposity is likely to result from both dysfunctional aSAT and VAT accumulation; this becomes clinically apparent among individuals with increased waist-to-hip ratio (WHR) $[17,18]$. Although accumulation of fat in aSAT is considered to be less detrimental than excessive VAT depot, disproportionate expansion of dSAT compared to sSAT has been associated with CVD risk factors; dSAT shares morphological and functional similarities to VAT $[7,19]$. However, these models might be too simplistic as attempts to remove VAT in randomized trials using surgical omentectomy do not appear to show any metabolic benefit [20-25].

Abdominal adiposity, particularly VAT, has been shown to be associated with dyslipidaemia characterised by raised concentrations of total and very-low-density lipoprotein (VLDL)triglycerides (TG), low high-density lipoprotein (HDL)-cholesterol, and an abundance of smaller and denser LDL particles despite relatively normal total and low-density lipoprotein (LDL)cholesterol levels [26]. This dyslipidaemic profile could be fuelled by excess flux of fatty acids (FA) to the liver. However, the relevance of the quantitative contribution of non-esterified fatty acid (NEFA) delivery from VAT has been questioned as it only accounts to $\sim 15 \%$ of the total systemic NEFA circulation, while majority of the NEFA is delivered by SAT [27, 28]. VAT is more metabolically active (greater fat storage and release) leading to higher lipid turnover than other fat tissues, greater lipolytic rates in response to stress hormones, and a lesser response to the anti-lipolytic effect of insulin, thus promoting atherogenic lipid and lipoprotein profiles compared 
to aSAT (Figure 1) [29]. Although it is suggested that the aSAT depot may help to regulate lipid metabolism by sequestering lipids destined to ectopic fat depots [30,31], the heterogeneity of the aSAT depot makes this questionable. The deeper aspect of the depot (dSAT) appears to have similarities to VAT [7], and an expansion of dSAT may therefore impact negatively on effective fat storage with effects on lipid and lipoprotein metabolism.

\section{Lower body gluteofemoral fat depots}

The CVD and diabetes-protective properties of the lower body gluteofemoral fat depots across a wide range of age, BMI and co-morbidities is well-established [11, 32-36]. Gluteofemoral fat, as measured by thigh circumference, HC or leg fat mass, is associated with lower total- and LDLcholesterol, lower total- and VLDL-TG levels, and higher HDL-cholesterol levels [37-40]. In the INTERHEART study, an independent association between larger $\mathrm{HC}$ and lower risk for myocardial infarction has been shown [9]. In the prospective European Prospective Investigation into Cancer and Nutrition-Norfolk study, larger HC was associated with a lower hazard ratio for CHD [8]. In a cross-sectional study involving 683 university students, higher levels of leg fat mass, as measured using DEXA, have been associated with a more favourable lipid profile, regardless of total body fatness, cardio-respiratory fitness or physical activity levels [41]. Change in leg fat mass after a 14-weeks intervention with diet and exercise was inversely associated with LDLcholesterol levels and a number of CVD risk factors in overweight and obese women [42]. The positive health effects of gluteofemoral fat could probably be related to its various inherent properties such as its ability to serve as a long-term storage reservoir for excess fat, thus acting as a "metabolic sink", and protecting other tissues from excessive exposure to lipids $[32,43]$. The tissue is characterised by a low lipid turnover compared with abdominal fat depots [30]. During energy deficit, the gluteofemoral depots shrink more slowly than abdominal depots [44-46]. Absence of gluteofemoral fat, such as in familial partial lipodystrophy, is almost invariably associated with dyslipidaemia and liver fat accumulation. 


\section{What is the evidence that fat depots handle lipid and lipoproteins differently?}

Factors that regulate lipid trafficking in fat depots is balanced between the rate of lipolysis and the rate of fat storage originating from chylomicron or VLDL-TG. There is also a small fraction of direct uptake of NEFA by adipose tissue (AT). The aSAT is characterised by a greater FA uptake from diet-derived FA and a high turnover that is easily stimulated by adrenergic receptor activation. In contrast, the gluteofemoral AT depots are characterised by a reduced turnover and a higher capacity to accommodate lipids undergoing redistribution (recirculating FA from VLDLTG or from NEFA pool) (Figure 1) [1]. The uptake of diet-derived FA occurs less efficiently in the gluteofemoral fat than in the abdominal fat depots $[28,47]$. This is balanced by less FA release from gluteofemoral AT in the postabsorptive state or during adrenergic lipolytic stimuli $[28,48]$. Direct measurements of lipid turnover after oral administration of radiolabelled FA show faster short-term uptake of diet-derived lipids in subcutaneous abdominal than in femoral fat depot [47, $49,50]$. Administration of isotopically labelled FA followed by serial biopsies of abdominal and femoral AT showed higher incorporation $(\sim 50 \%)$ of diet-derived FA in the abdominal than in the femoral fat depot [49]. Additional metabolic studies consistently demonstrated higher uptake of chylomicrons-derived FA in abdominal AT than in femoral AT [51-53].

Using two different FA labels to simultaneously quantify extraction of dietary chylomicronderived TG and VLDL-derived TG across abdominal and gluteofemoral AT depots, McQuaid and colleagues showed that chylomicron-derived TG were more efficiently extracted by abdominal AT, whereas no difference for VLDL-derived TG was observed [53]. Therefore, in relative terms the gluteofemoral AT depots have a preference for taking up FA from VLDL-TG compared with chylomicron-TG [53]. The extension of this observation is that VLDL-TG represents off-loading of lipids from the liver, which could suggest a hepato-femoral axis for fat storage. This would provide an explanation for the hepatic steatosis observed in patients lacking gluteofemoral AT depots, such as in familial partial lipodystrophy. 


\section{Sex difference in body fat distribution pattern}

The difference in body fat distribution between men and women has been related to both sexspecific lipid-lipoprotein profiles and CVD risk $[32,54]$. The female lower body fat depot is only reduced during periods of excessive energy demand [55]. After menopause, a switch in storage pattern from peripheral to central is observed and this is associated with a parallel increase in metabolic risks including adverse lipoprotein profile comparable to that seen in men [56]. However, an increase in total fat mass in men is rather immediately associated with accumulation in VAT [57]. Observations from transsexuals who have been treated with sex hormones have shown that the female-to-male transition induced by intramuscular testosterone injections show a progressive shift in body fat distribution from gluteofemoral to abdominal over a few months to 3 years [58-60]. Conversely, estrogen treatment in the male-to-female transition increases fat deposition in all SAT depots, while having little effect on the VAT depot [58, 60].

\section{$\underline{\text { Sex difference in fatty acid metabolism and metabolic consequences }}$}

In women, diet-derived lipid storage increases in proportion to lower body fat mass, while no association was found between relative lipid uptake in aSAT and adiposity measures [61]. In men, the capacity of aSAT to rapidly assimilate fat is higher compared with that of the femoral fat depot [62]. Indirect assessment of lipid uptake by fat depots suggests that VAT more efficiently removes lipids from the circulation during the postprandial period in men than in women $[47,63]$. Dietary FAs enter the circulation through chylomicron synthesis and may enter the liver in at least two ways [64]. FAs are released from TG-rich lipoprotein core of chylomicron by lipoprotein lipase. When this process occurs at a higher rate than the tissue uptake allows for, the excessive FAs spill over into the plasma NEFA pool and consequently contribute to the postprandial NEFA plasma concentrations reaching the liver. The chylomicron remnants with their remaining TG content are taken up by the liver. The FA spill over pathway is more pronounced in women compared to men, despite women having a greater fat mass [65]. This route could enhance recirculating FA 
toward long term lipid storage and there could also be different handling of the FAs in the liver depending on whether they enter as NEFA or by lipoprotein remnant uptake.

Sex differences in VLDL-TG metabolism have been reported in several studies [66, 67]. Women channelled a larger proportion of VLDL-TG to femoral AT depot for storage in the postabsorptive state [68]. Furthermore, the more femoral AT there is, the greater the efficiency to VLDL-TG uptake by this depot [69]. Men have higher plasma VLDL-TG concentrations and higher VLDL-TG secretion rates than women at any degree of adiposity [67]. Although hepatic VLDL-TG secretion rate was an important determinant of plasma VLDL-TG concentrations in both women and men, VLDL-TG secretion explained 70\% of VLDL-TG concentrations in plasma in men but only $30 \%$ in women, indicating that the female plasma TG concentrations are more reliant on catabolism.

\section{Adipose tissue expandability}

Upper and lower body AT respond differently to increasing demands for fat storage. Whilst the upper body subcutaneous AT depot displays a hyperplastic response (more fat in each fat cell), the gluteofemoral AT shows a proliferative response (new fat deposited in new fat cells) [70]. Expanding the fat stores in time of excess energy storage is a must to maintain metabolic health (Figure 2). The failure of AT expandability as a unifying hypothesis to explain complications of obesity was first muted by Eliot Danforth Jr [71]. Subsequently, this has been refined and underpinned by mechanistic insight by several groups $[72,73]$. More recently, this concept has been given support using large-scale genetic resources. Yaghootkar and colleagues have shown that carriers of 'favourable adiposity alleles' have a higher BMI and body fat percentage but, paradoxically, lower risk of diabetes, hypertension and CVD disease a phenotype that would be consistent with metabolically healthy obese, than non-carriers of these favourable adiposity alleles [2], suggesting that the absence of expandability leads to obesity-related complications. It is possible that these individuals are characterised by a more uniform fat distribution which is geared towards subcutaneous, rather than VAT expansion [74]. According to this hypothesis, the 
failure of appropriate AT expansion and fat storage leads to overflow of lipids which will be stored in ectopic non AT [75], which is usually the case in an obese individual characterised by greater VAT and lower peripheral subcutaneous fat. With this in mind the relationships between plasma lipids and regional AT depot masses becomes complex as there might be a direct positive relationship between VAT and raised lipids, whereas a lower subcutaneous AT mass is likely may either reflect leanness or a failure of expansion. The same duality is likely to exist for lower body fat depots.

\section{Effect on the liver}

Overall body fatness and abdominal visceral adiposity are both associated with liver fat accumulation, which in turn is associated with hepatic overproduction of VLDL-TG particles fuelling hypertriglyceridaemia [76]. Hepatic VLDL production is primarily substrate driven, with the most important substrates being NEFAs [77]. As the liver accumulates fat, there is overproduction of large VLDL particles [78]. NEFAs are taken up by the liver in proportion to their delivery rate [79]. The release of NEFAs from upper body SAT is a major determinant of systemic NEFA plasma concentrations, whereas VAT may contribute FAs specifically to the liver [80]. Thus, individuals with greater amounts of VAT are likely to have abnormalities in hepatic FA metabolism. Again, this relationship is more complex than anticipated and a dissociation between VAT volume and liver fat content has also been demonstrated [81]. 


\section{Conclusion}

The fat depots in the human body handle lipid storage and release differently and this impacts lipid and lipoprotein metabolism both directly and indirectly. Direct effects are seen on how various TG-rich lipoprotein species are handled differently by upper and lower body fat depots and also how the fat depots respond to lipolytic stimuli. Indirect effects, such as the impact on whole body fat storage capacity, is determined by body fat distribution, where the lower body fat depot appears to sequester excess fat better than upper body abdominal fat depots alleviating the formation of ectopic fat accumulation. Determinants of regional fat distribution, be it by genetic, epigenetic or hormonal influences, is a major determinant for lipid and lipoprotein metabolism with direct effects on CVD risk.

\section{$\underline{\text { Key points }}$}

- Susceptibility to obesity related metabolic complications is not mediated by overall body fatness, but is largely dependent on the body fat distribution and the ability to sufficiently expand fat depots.

- Upper body abdominal and lower body gluteofemoral fat depots exhibits opposing associations with risk of cardiovascular disease and adverse lipid and lipoprotein profiles.

- The opposite relationship of abdominal and gluteofemoral fat depots to cardiovascular risk reflects differential ways in which these fat depots handle lipid and lipoproteins.

- Sex differences exist in lipids handling, which may explain the different implications of body fat depots in obesity-related lipid-lipoprotein profile and metabolic diseases in men and women. 


\section{Acknowledgements}

The authors thank their colleagues who contributed to the data presented. They would like to thank previous and current fellows in their group who have developed protocol and conducted studies helping them to formulate the ideas contained in this review.

\section{Financial support and sponsorship}

MEP is recipient of a postdoctoral training fellowship grants from the Fonds de Recherche du Québec-Santé (FRQ-S) and the Heart and Lung Institute Foundation. FK is supported by the British Heart Foundation (RG/17/1/32663). SKV is supported by the Throne-Holst Foundation. FK has received support from the NIHR Oxford BRC Obesity, Diet and Lifestyle Theme for the Oxford Biobank.

\section{Conflict of Interest}

None 


\section{Figure legends}

Figure 1. Functional differences between adipose tissue (AT) depots. Abdominal AT (abdominal subcutaneous adipose tissue (ASAT) and visceral adipose tissue (VAT) depots) is the primary site for immediate storage of diet-derived fat. These fat depots have a high lipid turnover and undergo lipolysis in response to adrenergic stress stimuli. The gluteofemoral AT depot is characterised by a reduced lipid turnover with a high capacity to accommodate lipids undergoing redistribution consisting of recirculated fatty acids (from VLDL triglycerides or directly from the non-esterified fatty acid (NEFA) pool. The uptake of diet-derived fatty acids occurs less efficiently in the gluteofemoral fat than in the abdominal fat depot. This is balanced by less fatty acids release from gluteofemoral fat depot in the postabsorptive state or during adrenergic lipolytic stimuli. Consequently, this fat depot retains fatty acids well, which results in diminished exposure of ectopic tissues to lipids. AT, adipose tissue; VLDL, very low density lipoprotein; NEFA, nonesterified fatty acid

Figure 2. Relationship between body fat depots and obesity phenotypes against cardiovascular disease risk. Axis represent total fat percentage (total fat mass/total mass), visceral fat mass (VAT) and gluteofemoral fat mass (GF). Metabolically healthy normal weight (MHNW) individuals are characterised by relative $\uparrow$ GF and $\downarrow$ VAT; MHO (metabolically healthy obese) characterised by $\uparrow \uparrow$ GF; MUNW (metabolically unhealthy, normal weight) characterised by $\uparrow$ VAT; and MUO (metabolically unhealthy obese) characterised by $\downarrow$ GF and $\uparrow \uparrow$ VAT. At any given level of android fat, DEXA-measured peripheral fat (gynoid fat) is shown to be associate with reduced levels of fasting glucose, triglycerides, HOMA-IR (insulin resistance), and blood pressure. The differential association of upper and lower body fat depots with metabolic traits are evident in higher tertiles of fat mass index [11]. Thus, it is possible that such paradoxical associations are observed primarily in higher spectrum of obese phenotypes (MUNW and obese) characterized by greater total fat percentage. MHNW, metabolically healthy normal weight; MHO, metabolically healthy obese; MUNW, metabolically unhealthy normal weight; MUO, metabolically unhealthy obese. 


\section{$\underline{\text { References }}$}

[1] Karpe F, Pinnick KE. Biology of upper-body and lower-body adipose tissue--link to wholebody phenotypes. Nat Rev Endocrinol 2015; 11:90-100.

[2] Yaghootkar H, Lotta LA, Tyrrell J et al. Genetic Evidence for a Link Between Favorable Adiposity and Lower Risk of Type 2 Diabetes, Hypertension, and Heart Disease. Diabetes 2016; 65:2448-2460.

- This landmark paper provides independent genetic underpinning of the adipose tissue expandability hypothesis.

[3] Thomas EL, Saeed N, Hajnal JV et al. Magnetic resonance imaging of total body fat. J Appl Physiol (1985) 1998; 85:1778-1785.

[4] Kvist H, Ströström L, Tylen U. Adipose tissue volume determinations in women by computed tomography: technical considerations. Int J Obes 1986; 10:53-67.

[5] Johnson D, Dixon AK, Abrahams PH. The abdominal subcutaneous tissue: computed tomographic, magnetic resonance, and anatomical observations. Clin Anat 1996; 9:19-24.

[6] Lancerotto L, Stecco C, Macchi V et al. Layers of the abdominal wall: anatomical investigation of subcutaneous tissue and superficial fascia. Surg Radiol Anat 2011; 33:835-842.

[7] Marinou K, Hodson L, Vasan SK et al. Structural and functional properties of deep abdominal subcutaneous adipose tissue explain its association with insulin resistance and cardiovascular risk in men. Diabetes Care 2014; 37:821-829.

[8] Canoy D, Boekholdt SM, Wareham N et al. Body fat distribution and risk of coronary heart disease in men and women in the European Prospective Investigation Into Cancer and Nutrition in Norfolk cohort: a population-based prospective study. Circulation 2007; 116:2933-2943.

[9] Yusuf S, Hawken S, Ounpuu S et al. Obesity and the risk of myocardial infarction in 27,000 participants from 52 countries: a case-control study. Lancet 2005; 366:1640-1649.

[10] Snijder MB, Zimmet PZ, Visser M et al. Independent and opposite associations of waist and hip circumferences with diabetes, hypertension and dyslipidemia: the AusDiab Study. Int J Obes Relat Metab Disord 2004; 28:402-409.

[11] Vasan SK, Osmond C, Canoy D et al. Comparison of regional fat measurements by dual-energy X-ray absorptiometry and conventional anthropometry and their association with markers of diabetes and cardiovascular disease risk. Int J Obes (Lond) 2017. doi: 10.1038/ijo.2017.289.

- This publication describes how precise quantification of regional fat masses enhances the ability to detect the paradoxical relationships between upper and lower body adiposity and obesity-related consequences.

[12] Fox CS, Massaro JM, Hoffmann U et al. Abdominal visceral and subcutaneous adipose tissue compartments: association with metabolic risk factors in the Framingham Heart Study. Circulation 2007; 116:39-48.

[13] Liu J, Fox CS, Hickson DA et al. Impact of abdominal visceral and subcutaneous adipose tissue on cardiometabolic risk factors: the Jackson Heart Study. J Clin Endocrinol Metab 2010; 95:54195426.

[14] Preis SR, Massaro JM, Robins SJ et al. Abdominal subcutaneous and visceral adipose tissue and insulin resistance in the Framingham Heart Study. Obesity (Silver Spring) 2010; 18:21912198.

[15] Abraham TM, Pedley A, Massaro JM et al. Association between visceral and subcutaneous adipose depots and incident cardiovascular disease risk factors. Circulation 2015; 132:16391647.

[16] Fujimoto WY, Bergstrom RW, Boyko EJ et al. Visceral adiposity and incident coronary heart disease in Japanese-American men. The 10-year follow-up results of the Seattle JapaneseAmerican Community Diabetes Study. Diabetes Care 1999; 22:1808-1812. 
[17] Sam S, Haffner S, Davidson MH et al. Relationship of abdominal visceral and subcutaneous adipose tissue with lipoprotein particle number and size in type 2 diabetes. Diabetes 2008; 57:2022-2027.

[18] Abate N, Garg A, Peshock RM et al. Relationships of generalized and regional adiposity to insulin sensitivity in men. J Clin Invest 1995; 96:88-98.

[19] Kelley DE, Thaete FL, Troost F et al. Subdivisions of subcutaneous abdominal adipose tissue and insulin resistance. Am J Physiol Endocrinol Metab 2000; 278:E941-948.

[20] Andersson DP, Eriksson-Hogling D, Backdahl J et al. Omentectomy in Addition to Bariatric Surgery-a 5-Year Follow-up. Obes Surg 2017; 27:1115-1118.

[21] Fabbrini E, Tamboli RA, Magkos F et al. Surgical removal of omental fat does not improve insulin sensitivity and cardiovascular risk factors in obese adults. Gastroenterology 2010; 139:448-455.

[22] Andersson DP, Thorell A, Lofgren P et al. Omentectomy in addition to gastric bypass surgery and influence on insulin sensitivity: a randomized double blind controlled trial. Clin Nutr 2014; 33:991-996.

[23] Sdralis E, Argentou M, Mead N et al. A prospective randomized study comparing patients with morbid obesity submitted to sleeve gastrectomy with or without omentectomy. Obes Surg 2013; 23:965-971.

[24] Dunn JP, Abumrad NN, Breitman I et al. Hepatic and peripheral insulin sensitivity and diabetes remission at 1 month after Roux-en-Y gastric bypass surgery in patients randomized to omentectomy. Diabetes Care 2012; 35:137-142.

[25] Csendes A, Maluenda F, Burgos AM. A prospective randomized study comparing patients with morbid obesity submitted to laparotomic gastric bypass with or without omentectomy. Obes Surg 2009; 19:490-494.

[26] Pouliot MC, Després JP, Nadeau A et al. Visceral obesity in men. Associations with glucose tolerance, plasma insulin, and lipoprotein levels. Diabetes 1992; 41:826-834.

[27] Garg A. Regional adiposity and insulin resistance. J Clin Endocrinol Metab 2004; 89:42064210.

[28] Jensen MD, Johnson CM. Contribution of leg and splanchnic free fatty acid (FFA) kinetics to postabsorptive FFA flux in men and women. Metabolism 1996; 45:662-666.

[29] Arner P. Differences in lipolysis between human subcutaneous and omental adipose tissues. Ann Med 1995; 27:435-438.

[30] Frayn KN. Adipose tissue as a buffer for daily lipid flux. Diabetologia 2002; 45:1201-1210.

[31] Golan R, Shelef I, Rudich A et al. Abdominal superficial subcutaneous fat: a putative distinct protective fat subdepot in type 2 diabetes. Diabetes Care 2012; 35:640-647.

[32] Manolopoulos KN, Karpe F, Frayn KN. Gluteofemoral body fat as a determinant of metabolic health. Int J Obes (Lond) 2010; 34:949-959.

[33] Terry RB, Stefanick ML, Haskell WL, Wood PD. Contributions of regional adipose tissue depots to plasma lipoprotein concentrations in overweight men and women: possible protective effects of thigh fat. Metabolism 1991; 40:733-740.

[34] Choi SI, Chung D, Lim JS et al. Relationship between Regional Body Fat Distribution and Diabetes Mellitus: 2008 to 2010 Korean National Health and Nutrition Examination Surveys. Diabetes Metab J 2017; 41:51-59.

- This publication presents regional body fat distribution data, including leg fat mass, measures using DEXA, in men and postmenopausal women in regard to the prevalence of diabetes mellitus. This study found that higher leg fat mass was independently associated with a lower risk of diabetes mellitus in adult populations.

[35] Sakai Y, Ito H, Egami Y et al. Favourable association of leg fat with cardiovascular risk factors. J Intern Med 2005; 257:194-200.

[36] Wiklund P, Toss F, Jansson JH et al. Abdominal and gynoid adipose distribution and incident myocardial infarction in women and men. Int J Obes (Lond) 2010; 34:1752-1758. 
[37] Seidell JC, Pérusse L, Després JP, Bouchard C. Waist and hip circumferences have independent and opposite effects on cardiovascular disease risk factors: the Quebec Family Study. Am J Clin Nutr 2001; 74:315-321.

[38] Snijder MB, Visser M, Dekker JM et al. Low subcutaneous thigh fat is a risk factor for unfavourable glucose and lipid levels, independently of high abdominal fat. The Health ABC Study. Diabetologia 2005; 48:301-308.

[39] Williams MJ, Hunter GR, Kekes-Szabo T et al. Regional fat distribution in women and risk of cardiovascular disease. Am J Clin Nutr 1997; 65:855-860.

[40] Yim JE, Heshka S, Albu JB et al. Femoral-gluteal subcutaneous and intermuscular adipose tissues have independent and opposing relationships with CVD risk. J Appl Physiol (1985) 2008; 104:700-707.

[41] Sanchez-Lopez M, Ortega FB, Moya-Martinez P et al. Leg fat might be more protective than arm fat in relation to lipid profile. Eur J Nutr 2013; 52:489-495.

[42] Okura T, Nakata Y, Yamabuki K, Tanaka K. Regional body composition changes exhibit opposing effects on coronary heart disease risk factors. Arterioscler Thromb Vasc Biol 2004; 24:923-929.

[43] Lemieux I. Energy partitioning in gluteal-femoral fat: does the metabolic fate of triglycerides affect coronary heart disease risk? Arterioscler Thromb Vasc Biol 2004; 24:795-797.

[44] Jones PR, Edwards DA. Areas of fat loss in overweight young females following an 8-week period of energy intake reduction. Ann Hum Biol 1999; 26:151-162.

[45] Goodpaster BH, Kelley DE, Wing RR et al. Effects of weight loss on regional fat distribution and insulin sensitivity in obesity. Diabetes 1999; 48:839-847.

[46] Santosa S, Hensrud DD, Votruba SB, Jensen MD. The influence of sex and obesity phenotype on meal fatty acid metabolism before and after weight loss. Am J Clin Nutr 2008; 88:1134-1141.

[47] Romanski SA, Nelson RM, Jensen MD. Meal fatty acid uptake in adipose tissue: gender effects in nonobese humans. Am J Physiol Endocrinol Metab 2000; 279:E455-462.

[48] Manolopoulos KN, Karpe F, Frayn KN. Marked resistance of femoral adipose tissue blood flow and lipolysis to adrenaline in vivo. Diabetologia 2012; 55:3029-3037.

[49] Marin P, Rebuffe-Scrive M, Bjorntorp P. Uptake of triglyceride fatty acids in adipose tissue in vivo in man. Eur J Clin Invest 1990; 20:158-165.

[50] Jensen MD, Sarr MG, Dumesic DA et al. Regional uptake of meal fatty acids in humans. Am J Physiol Endocrinol Metab 2003; 285:E1282-1288.

[51] Jensen MD. Gender differences in regional fatty acid metabolism before and after meal ingestion. J Clin Invest 1995; 96:2297-2303.

[52] Guo Z, Johnson CM, Jensen MD. Regional lipolytic responses to isoproterenol in women. Am J Physiol 1997; 273:E108-112.

[53] McQuaid SE, Humphreys SM, Hodson L et al. Femoral adipose tissue may accumulate the fat that has been recycled as VLDL and nonesterified fatty acids. Diabetes 2010; 59:2465-2473.

[54] Tchernof A, Després JP. Pathophysiology of human visceral obesity: an update. Physiol Rev 2013; 93:359-404.

[55] Kramer FM, Stunkard AJ, Marshall KA et al. Breast-feeding reduces maternal lower-body fat. J Am Diet Assoc 1993; 93:429-433.

[56] Shimokata H, Tobin JD, Muller DC et al. Studies in the distribution of body fat: I. Effects of age, sex, and obesity. J Gerontol 1989; 44:M66-73.

[57] Lemieux S, Prud'homme D, Bouchard C et al. Sex differences in the relation of visceral adipose tissue accumulation to total body fatness. Am J Clin Nutr 1993; 58:463-467.

[58] Elbers JM, Asscheman H, Seidell JC, Gooren LJ. Effects of sex steroid hormones on regional fat depots as assessed by magnetic resonance imaging in transsexuals. Am J Physiol 1999; 276:E317325.

[59] Elbers JM, Asscheman H, Seidell JC et al. Long-term testosterone administration increases visceral fat in female to male transsexuals. J Clin Endocrinol Metab 1997; 82:2044-2047.

[60] Elbers JM, Giltay EJ, Teerlink T et al. Effects of sex steroids on components of the insulin resistance syndrome in transsexual subjects. Clin Endocrinol (Oxf) 2003; 58:562-571. 
[61] Koutsari C, Dumesic DA, Patterson BW et al. Plasma free fatty acid storage in subcutaneous and visceral adipose tissue in postabsorptive women. Diabetes 2008; 57:1186-1194.

[62] Shadid S, Koutsari C, Jensen MD. Direct free fatty acid uptake into human adipocytes in vivo: relation to body fat distribution. Diabetes 2007; 56:1369-1375.

[63] Nguyen TT, Hernandez Mijares A, Johnson CM, Jensen MD. Postprandial leg and splanchnic fatty acid metabolism in nonobese men and women. Am J Physiol 1996; 271:E965-972.

[64] Barrows BR, Parks EJ. Contributions of different fatty acid sources to very low-density lipoprotein-triacylglycerol in the fasted and fed states. J Clin Endocrinol Metab 2006; 91:14461452.

[65] Piche ME, Parry SA, Karpe F, Hodson L. Chylomicron-Derived Fatty Acid Spillover in Adipose Tissue: A Signature of Metabolic Health? J Clin Endocrinol Metab 2018; 103:25-34.

- This paper makes use of sophisticated in vivo physiology with stable isotope labelled fatty acid tracers to describe the generation of the fatty spillover pathway in human adipose tissue. It appears to be a physiologically normal phenomenon which is augmented in people with high lipoprotein lipase action.

[66] Magkos F, Patterson BW, Mohammed BS et al. Women produce fewer but triglyceride-richer very low-density lipoproteins than men. J Clin Endocrinol Metab 2007; 92:1311-1318.

[67] Mittendorfer B, Yoshino M, Patterson BW, Klein S. VLDL Triglyceride Kinetics in Lean, Overweight, and Obese Men and Women. J Clin Endocrinol Metab 2016; 101:4151-4160.

- In this study, the authors used sophisticated stable isotope labeled tracer techniques to evaluate factors modulating hepatic VLDL metabolism in a large cohort of men and women with a wide range in body adiposity and fat distribution. It was found that substrate availability for VLDL-TG synthesis is a major driver for VLDL-TG secretion, particularly free fatty acid derived from adipose tissue lipolysis and intrahepatic triglycerides.

[68] Gormsen LC, Nellemann B, Sorensen LP et al. Impact of body composition on very-lowdensity lipoprotein-triglycerides kinetics. Am J Physiol Endocrinol Metab 2009; 296:E165-173.

[69] Nellemann B, Gormsen LC, Christiansen JS et al. Postabsorptive VLDL-TG fatty acid storage in adipose tissue in lean and obese women. Obesity (Silver Spring) 2010; 18:1304-1311.

[70] Tchoukalova YD, Votruba SB, Tchkonia T et al. Regional differences in cellular mechanisms of adipose tissue gain with overfeeding. Proc Natl Acad Sci U S A 2010; 107:18226-18231.

[71] Danforth E, Jr. Failure of adipocyte differentiation causes type II diabetes mellitus? Nat Genet $2000 ; 26: 13$.

[72] Virtue S, Vidal-Puig A. Adipose tissue expandability, lipotoxicity and the Metabolic Syndrome--an allostatic perspective. Biochim Biophys Acta 2010; 1801:338-349.

[73] Taylor R, Holman RR. Normal weight individuals who develop type 2 diabetes: the personal fat threshold. Clin Sci (Lond) 2015; 128:405-410.

[74] Vasan SK, Karpe F. Adipose tissue: Fat, yet fit. Nat Rev Endocrinol 2016; 12:375-376.

[75] McQuaid SE, Hodson L, Neville MJ et al. Downregulation of adipose tissue fatty acid trafficking in obesity: a driver for ectopic fat deposition? Diabetes 2011; 60:47-55.

[76] Adiels M, Taskinen MR, Packard C et al. Overproduction of large VLDL particles is driven by increased liver fat content in man. Diabetologia 2006; 49:755-765.

[77] Lewis GF. Fatty acid regulation of very low density lipoprotein production. Curr Opin Lipidol 1997; 8:146-153.

[78] Fabbrini E, Mohammed BS, Magkos F et al. Alterations in adipose tissue and hepatic lipid kinetics in obese men and women with nonalcoholic fatty liver disease. Gastroenterology 2008; 134:424-431.

[79] Wahren J, Sato Y, Ostman J et al. Turnover and splanchnic metabolism of free fatty acids and ketones in insulin-dependent diabetics at rest and in response to exercise. J Clin Invest 1984; 73:1367-1376.

[80] Nielsen S, Guo Z, Johnson CM et al. Splanchnic lipolysis in human obesity. J Clin Invest 2004; 113:1582-1588. 
[81] Fabbrini E, Magkos F, Mohammed BS et al. Intrahepatic fat, not visceral fat, is linked with metabolic complications of obesity. Proc Natl Acad Sci U S A 2009; 106:15430-15435. 


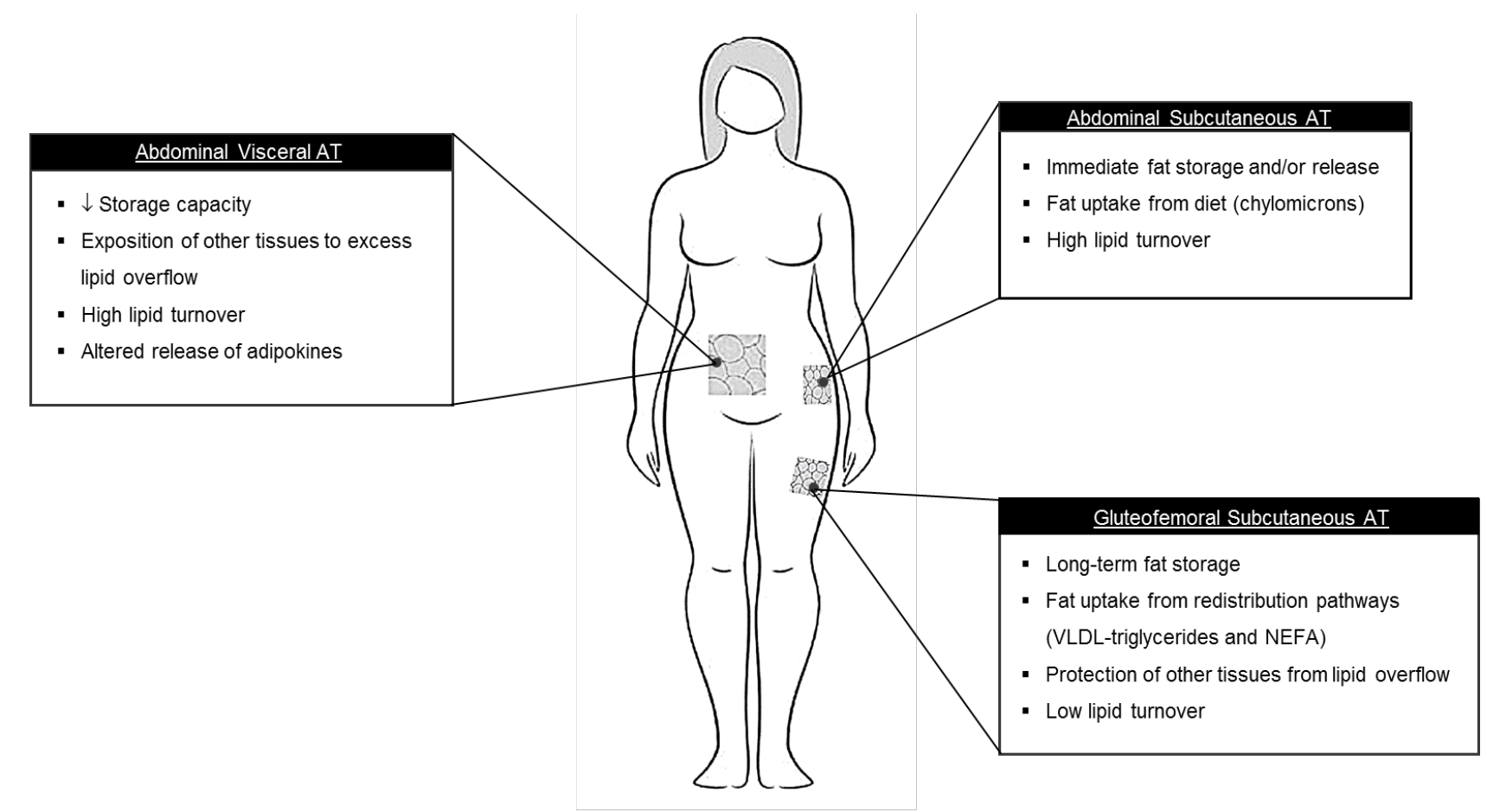

Figure 1 Functional differences between adipose tissue depots 


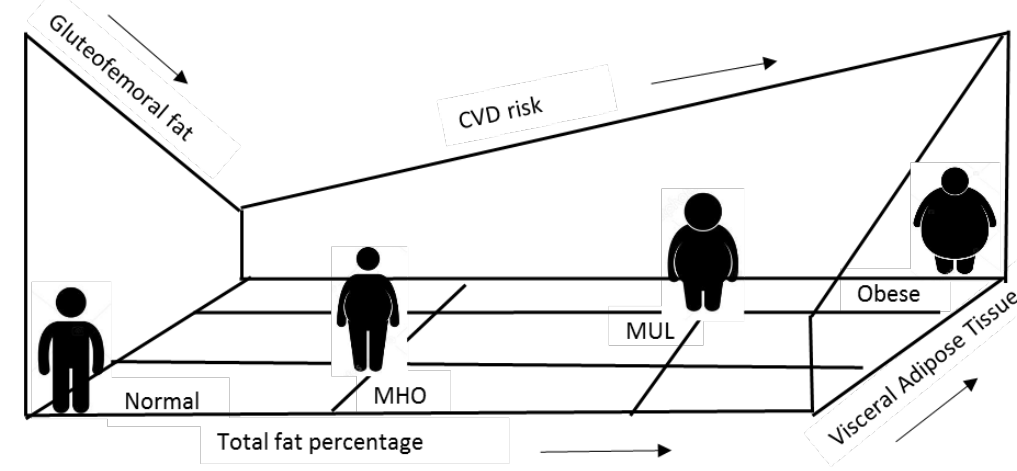

Figure 2. Relationship between body fat depots and obesity phenotypes against cardiovascular risk. Axis represent Total fat percentage (total fat mass/total mass), visceral adipose tissue (VAT) and Gluteofemoral fat (GF). Normal individuals are characterised by $\uparrow$ GF and $\downarrow$ VAT; MHO (metabolically healthy obese characterised by $\uparrow \uparrow \mathrm{GF}, \mathrm{MUL}$ (metabolically healthy, but lean) characterised by $\uparrow \uparrow \mathrm{VAT}$ and obese phenotype characterised by $\downarrow$ GF and $\uparrow \uparrow \mathrm{VAT}$. 